\title{
Glycated hemoglobin level is significantly associated with the severity of coronary artery disease in non-diabetic adults
}

\author{
Anping Cai ${ }^{\dagger}$, Guang Li ${ }^{\dagger}$, Jiyan Chen, Xida Li, Xuebiao Wei, Liwen Li ${ }^{*}$ and Yingling Zhou
}

\begin{abstract}
Background: To investigate relationship between glycated hemoglobin ( $\mathrm{HbA1c}$ ) level and coronary artery disease (CAD) severity.

Methods: Observational study was conducted and 573 participants were enrolled and baseline characteristics were collected. Clinical presentations in terms of stable angina, unstable angina or acute myocardial infarction were diagnosed. All participants were performed coronary angiography to figure out the numbers of coronary artery stenosis in terms of none-stenosis ( $<50 \%$ stenosis), single or multiple vessels stenoses ( $\geq 50 \%$ stenosis). All participants were divided into subgroups according to two categories in terms of severity of clinical presentation (stable angina, unstable angina, or acute myocardial infarction) and the number of coronary artery stenosis (none, single, and multiple vessels). Primary endpoint was to evaluate relationship between baseline $\mathrm{HbA1c}$ value and CAD severity.
\end{abstract}

Results: Consistent to previous studies, participants with CAD had more risk factors such as elderly, smoking, low HDL-C and high CRP levels. Notably, HbA1c level was more prominent in CAD group than that without CAD. As compared to stable angina subgroup, HbA1c levels were gradually increased in unstable angina and acute myocardial infarction groups. Similar trend was identified in another category in terms of higher HbA1c level corresponding to more vessels stenoses. Multivariate regression analyses showed that after adjusted for traditional risk factors as well as fasting blood glucose, HbA1c remained strongly associated with the severity of CAD. Nonetheless, there was no significant association when CRP was accounted for.

Conclusion: $\mathrm{HbA} 1 \mathrm{c}$ may be a useful indicator for CAD risk evaluation in non-diabetic adults.

Keywords: Glycated hemoglobin, Coronary artery disease, Diabetes mellitus

\section{Introduction}

Diabetes mellitus is a major and well established risk factor for macro-vascular diseases such as atherosclerotic cardiovascular diseases (ASCVD) and micro-vascular diseases such as neural, renal and retinal diseases $[1,2]$. Of note, in patients with diabetes, plasma glucose level is significantly associated with the incidence and severity of micro-vascular and macro-vascular complications [1]. Currently, owing to its advantages over fasting blood glucose such as low intra-individual variability and being

\footnotetext{
* Correspondence: gdghllw@163.com

${ }^{\dagger}$ Equal contributors

Department of Cardiology, Guangdong Cardiovascular Institute, Guangdong

General Hospital, Guangdong Academy of Medical Sciences, 106 Zhongshan Road 2, Guangzhou 510080, China
}

(c) 2014 Cai et al.; licensee BioMed Central Ltd. This is an Open Access article distributed under the terms of the Creative

capable of evaluating the long-term blood glucose control [3,4], glycated hemoglobin (HbA1c), a parameter of average blood glucose levels over 12 weeks, has also been suggested using in clinical practice currently [5,6]. Evidence from epidemiological studies also showed that as compared to fasting blood glucose, HbA1c was more strongly associated with the risks of ASCVD and mortality from any causes $[7,8]$, which further supported the notion that HbA1c was superior to fasting blood glucose in predicting ASCVD outcomes. Nevertheless, some drawbacks regarding $\mathrm{HbA1c}$, including un-satisfactory correlation with fasting blood glucose and over-diagnosis among patients with anemia and renal dysfunction, have also aroused concerns with respect to its application for diabetic diagnosis and 
ASCVD risk prediction $[9,10]$. And the truly relationship between HbA1c and cardiovascular outcomes in populations without diabetes are still uncertain. For example, Silbernagel et al. reported that HbA1c significantly and independently of fasting blood glucose predicted all-cause of cardiovascular death in white population without diabetes [7]. Garg N and colleagues showed that in non-diabetics, HbA1c level has a linear incremental association with ASCVD [11]. Nonetheless, data from the Emerging Risk Factors Collaboration revealed that HbA1c merely added little incremental benefit for ASCVD risk prediction in patients without known ASCVD and diabetes [12].

In light of previous findings, our current study was designed to evaluate whether HbA1c level was associated with the severity of coronary artery diseases (CAD) in populations without diagnostic diabetes, and we believed that the clinical implication of our study would add, if any, valuable information to address whether $\mathrm{HbA1c}$ level could be used to predict the ASCVD risk in non-diabetic population.

\section{Methods}

\section{Studied populations and protocols}

Studied populations were enrolled from June of 2013 to April of 2014 after informed consent was obtained, and this study was approved by the Ethical Committee of Guangdong General Hospital. Current research is a retrospective and observational research, therefore, the including criteria are subjects with definitive coronary artery condition in terms of none-significant, single or multiple vessels stenoses as assessed by angiographic examination, and the excluding criteria are subjects without angiographic examination, with significant hepatic or renal dysfunction, anemia, tumor or connective tissue diseases. Clinical presentations in terms of stable angina, unstable angina or acute myocardial infarction were diagnosed according to their clinical manifestation, electrocardiography characteristics and cardiac biomarkers by two experts of cardiology. All participants were performed coronary angiography to definitely figure out the numbers of coronary artery stenosis in terms of none-significant $(<50 \%$ stenosis), single or multiple vessels stenoses ( $\geq 50 \%$ stenosis). Baseline characteristics including age, gender, family history of ASCVD, smoking status, hypertension, serum levels of lipid profile, fasting blood glucose, C-reactive protein (CRP) and HbA1c were collected at admission. In order to better understand whether HbA1c level was associated with the severity of CAD, all participants were divided into different subgroups according to two major categories in terms of the severity of clinical presentation (stable angina, unstable angina, or acute myocardial infarction) and the number of coronary artery stenosis (none, single, and multiple vessels stenoses).

\section{Studied endpoint}

The primary endpoint of current study was to evaluate the relationship between $\mathrm{HbA1c}$ level and the severity of CAD. In addition, whether HbA1c was an independent risk indicator for the severity of CAD was also evaluated.

\section{Statistical analyses}

Continuous data was presented as mean \pm SD or median (interquartile range) appropriately, and compared by the Student's t-test when data was normally distributed, otherwise compared by the Wilcoxon rank-sum test. Categorical data was presented as percentage and compared by $x^{2}$ test. Univariate and multivariate regression analyses were performed to evaluate the relationship between $\mathrm{HbA1c}$ value and the severity of coronary artery diseases. Statistical analyses were performed by using SPSS software version 16.0 (SPSS, Inc., Chicago, Illinois). A value of $\mathrm{p}<0.05$ was considered significant.

\section{Results}

\section{Baseline characteristics}

Totally 573 participants were enrolled and initially divided into two groups, namely without CAD group and CAD group, according to their coronary angiography examination. Since our current study was an observational study and therefore it was understandable and rational that the risk factors or comorbidities were more prevalent in the CAD group than that of without CAD group (as presented in Table 1). In the CAD group, age was older, male predominance, higher percentage of smoking and lower high density lipoprotein-cholesterol (HDL-C) level. No significant difference of total cholesterol (TC), triglyceride (TG), low density lipoprotein-cholesterol (LDL-C), renal function and fasting blood glucose were found between these two groups, except serum levels of CRP and HbA1c were significantly higher in the CAD group than that of without CAD group. Baseline characteristics among clinical subgroups were compared additionally. As presented in Table 2, risk factors including aging and smoking were more prevalent in the acute myocardial infarction group as compared to the stable angina group $(\mathrm{p}<0.05)$. Serum level of CRP was significantly higher while apoA level was profoundly lower in the acute myocardial infarction when compared to the table angina group $(\mathrm{p}<0.05)$.

\section{Comparison of $\mathrm{HbA1c}$ levels among subgroups of CAD}

In order to evaluate the relationship of HbA1c level and severity of CAD, all CAD patients $(n=466)$ were divided into different subgroups according to two major categories as described above. In the first category, all CAD patients were divided into stable angina, unstable angina and acute myocardial infarction groups. As shown in Figure 1A, the difference of HbA1c level among each subgroup was significantly different, with a $\mathrm{p}$-trend value $<0.05$. In the 
Table 1 Baseline characteristics

\begin{tabular}{llll}
\hline Variable & Without CAD $(\mathbf{n}=\mathbf{1 0 7})$ & CAD $(\mathbf{n}=\mathbf{4 6 6})$ & P value \\
\hline Age (years) & $60.5 \pm 12.1$ & $63.83 \pm 11.0$ & 0.006 \\
Male (\%) & $66(61.7)$ & $355(76.2)$ & 0.002 \\
Family history (\%) & $5(4.7)$ & $32(6.9)$ & 0.405 \\
Smoking (\%) & $27(25.2)$ & $173(37.1)$ & 0.020 \\
Hypertension (\%) & $50(46.7)$ & $273(58.6)$ & 0.260 \\
TG (mmol/L) & $1.51 \pm 1.05$ & $1.78 \pm 1.50$ & 0.078 \\
TC (mmol/L) & $4.44 \pm 1.01$ & $4.30 \pm 1.13$ & 0.264 \\
LDL-C (mmol/L) & $2.67 \pm 0.87$ & $2.62 \pm 0.99$ & 0.609 \\
HDL-C (mmol/L) & $1.11 \pm 0.30$ & $0.97 \pm 0.23$ & $<0.001$ \\
ApoA (mmol/L) & $1.24 \pm 0.39$ & $1.10 \pm 0.31$ & $<0.001$ \\
ApoB (mmol/L) & $0.81 \pm 0.60$ & $0.77 \pm 0.23$ & 0.273 \\
CRP (mg/L) & $3.67 \pm 0.60$ & $12.12 \pm 1.21$ & 0.001 \\
Cr (umol/L) & $96.5 \pm 10.1$ & $97.2 \pm 8.8$ & 0.228 \\
BUN (mmol/L) & $6.60 \pm 1.33$ & $6.82 \pm 1.07$ & 0.164 \\
FBG (mmol/L) & $5.58 \pm 0.76$ & $5.26 \pm 0.69$ & 0.178 \\
HbA1c (\%) & $6.05 \pm 1.17$ & $6.55 \pm 1.38$ & 0.001 \\
\hline
\end{tabular}

Denote: $\mathrm{TG}=$ triglyceride, $\mathrm{TC}=$ total cholesterol, $\mathrm{LDL}-\mathrm{C}=$ low density lipoprotein-cholesterol, HDL-C = high density lipoprotein-cholesterol, $\mathrm{Cr}=$ creatinine, $\mathrm{BUN}=$ blood urine nitrogen.

second category, all CAD patients were divided into nonesignificant stenosis, single vessel stenosis and multiple vessels stenoses groups. As presented in Figure 1B, the HbA1c level was significantly associated with the number of coronary artery stenosis, with a p-trend value $<0.001$.

\section{Multivariate regression analyses}

For the purpose of investigating whether HbA1c was an independent risk factor for the severity of CAD, multivariate regression analyses were performed. In the first category, after adjustment for age, gender, family history of CAD, smoking and hypertension (in model 1a), HbA1c was strongly associated with the severity of $\mathrm{CAD}$ ( $\mathrm{P}$ for trend $=$ 0.010). Additional adjustment for TC, HDL-C, ApoA, and fasting blood glucose, the association was attenuated but HbA1c remained significantly associated with the severity of CAD ( $\mathrm{P}$ for trend $=0.043$, in model $1 \mathrm{~b}$ ). Notably, with adjustment for CRP ( $\mathrm{P}$ for trend $=0.265$, in model $1 \mathrm{c}$ ), there was no significant association between $\mathrm{HbA1c}$ and the severity of CAD. In the second category, after adjustment for age, gender, family history of CAD, smoking and hypertension (in model 2a), there was a strongly relationship between $\mathrm{HbA1c}$ and the number of coronary artery stenosis ( $\mathrm{P}$ for trend $<0.001$ ). Additional adjustment for TC, HDL-C, ApoA, and fasting blood glucose (in model $2 \mathrm{~b}), \mathrm{HbA} 1 \mathrm{c}$ remained strongly associated with the number of coronary artery stenosis (P for trend $<0.001)$. Similarly, with adjustment for CRP ( $\mathrm{P}$ for trend $=0.168$, in model $2 \mathrm{c}$ ), there was no significant association between HbA1c and the number of coronary artery stenosis.

\section{Discussion}

Currently, the predictive value of HbA1c on ASCVD risk in non-diabetic population is still less certain, and the application of HbA1c on ASCVD risk assessment among different Cardiovascular Society is also still inconsistent. Findings from our current study show that there is a significant association between HbA1c level and the severity of CAD in non-diabetic population, even after adjustment for traditional risk factors, and this relationship is independent of fasting blood glucose. Nonetheless, we also observed that after adjustment for CRP,

Table 2 Comparisons of baseline characteristics of clinical sub-groups

\begin{tabular}{lllll}
\hline Variable & None $(\mathbf{n}=\mathbf{1 2 9})$ & Single $(\mathbf{n}=\mathbf{8 7})$ & Multi $(\mathbf{n}=\mathbf{2 5 0})$ & $\mathbf{P}$ value \\
\hline Age (years) & $59.7 \pm 10.4$ & $61.6 \pm 11.3$ & $64.2 \pm 9.7$ & $355(80.4)$ \\
Male (\%) & $90(69.8)$ & $64(73.6)$ & $32(7.2)$ & $<.024$ \\
Family history (\%) & $8(6.2)$ & $6(6.9)$ & $99(39.6)$ & 0.001 \\
Smoking (\%) & $41(31.8)$ & $33(37.9)$ & $149(59.6)$ & 0.113 \\
Hypertension (\%) & $72(55.8)$ & $52(59.8)$ & $1.73 \pm 0.22$ & 0.036 \\
TG (mmol/L) & $1.62 \pm 0.21$ & $1.71 \pm 0.18$ & $4.70 \pm 1.05$ & 0.218 \\
TC (mmol/L) & $4.65 \pm 1.02$ & $4.59 \pm 1.01$ & $2.86 \pm 0.57$ & 0.133 \\
LDL-C (mmol/L) & $2.82 \pm 0.62$ & $2.84 \pm 0.55$ & $0.95 \pm 0.20$ & 0.428 \\
HDL-C (mmol/L) & $1.08 \pm 0.26$ & $0.99 \pm 0.24$ & $1.13 \pm 0.43$ & 0.550 \\
APOA (mmol/L) & $1.29 \pm 0.41$ & $1.22 \pm 0.36$ & $0.80 \pm 0.47$ & 0.446 \\
APOB (mmol/L) & $0.82 \pm 0.56$ & $0.80 \pm 0.35$ & $13.65 \pm 1.17$ & 0.023 \\
CRP (mg/L) & $9.24 \pm 1.08$ & $10.86 \pm 1.12$ & $97.6 \pm 8.2$ & 0.204 \\
Cr (umol/L) & $96.8 \pm 8.1$ & $97.0 \pm 6.4$ & $6.82 \pm 1.03$ & 0.523 \\
BUN (mmol/L) & $6.67 \pm 1.03$ & $6.80 \pm 1.01$ & $5.29 \pm 0.62$ & 0.667 \\
FBG (mmol/L) & $5.22 \pm 0.44$ & $5.26 \pm 0.38$ & 0.216 \\
\hline
\end{tabular}



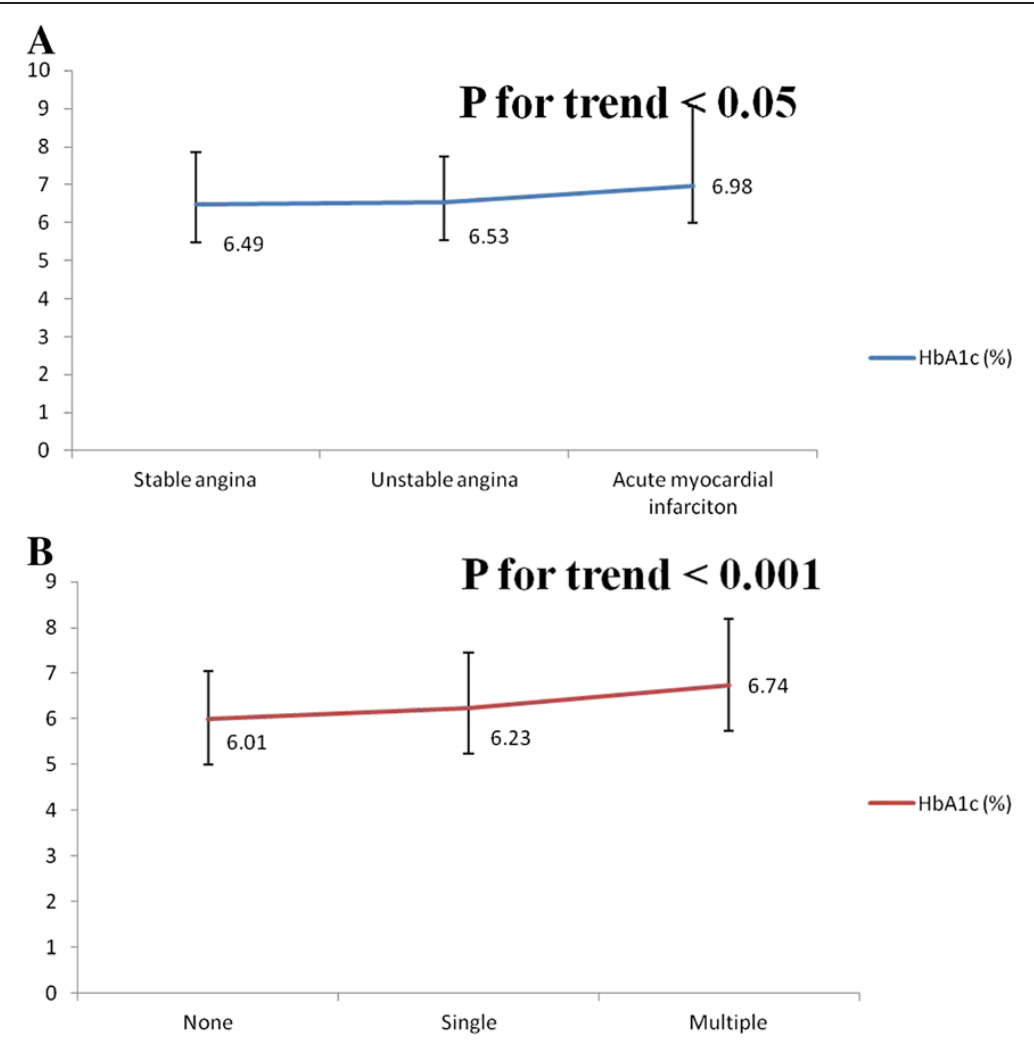

Figure 1 Comparison of HbA1c levels among each subgroup in the two categories. Panel A, HbA1c levels comparison among stable, unstable and AMl groups. Panel B, HbA1c levels comparison among none, single, and multiple vessels stenoses groups.

there was no significant association between $\mathrm{HbA} 1 \mathrm{c}$ and the severity of CAD.

Knowingly, glycated hemoglobin is a stable parameter reflecting approximately 3 months of blood glucose levels and it has now been suggested using in clinical practice for long-term blood glucose assessment in diabetic patients $[1,13]$. HbA1c is calculated as the ratio of glycated to non-glycated $\mathrm{N}$-terminal peptide of hemoglobin, and the HbA1c level has been universally recognized as important indicator for micro-vascular complications in diabetic patients [13,14]. Nevertheless, whether HbA1c level could also be used in macro-vascular diseases (such as ASCVD) risk prediction is less clear, especially in nondiabetic populations $[15,16]$.

Accordingly, ASCVD is the leading cause of morbidity and mortality worldwide and improvement of risk discrimination is imperative [17]. Previously, results from previous studies showed that for coronary heart diseases, risk discrimination was significantly enhanced when $\mathrm{HbA1c}$ was added in risk algorithm in non-diabetic patients $[8,18,19]$. Our current study also showed that as compared to stable episode (stable angina), the levels of HbA1c were gradually and significantly increased in unstable conditions in terms of unstable angina and acute myocardial infarction, indicating that increased $\mathrm{HbA1c}$ level might result in atherosclerotic plaque pro-rupture, and to our best knowledge there were some mechanisms might partially explain this finding. Biologically, glycated hemoglobin is an advanced glycosylation end-product, and increased HbA1c level could reflect more generation of advanced glycosylation end-product, which might subsequently abundantly attached to vessel wall causing endothelial dysfunction and oxidative stress promotion $[20,21]$. On the other hand, the binding of advanced glycosylation end-product might also result in inflammatory cytokines such as CRP over-production [22]. Increased CRP level has been found significantly associated with the instability of plaque $[23,24]$, and this might partially explain why after adjustment for CRP, there was no significant association between HbA1c and the severity of CAD. Finally, increased advanced glycosylation end-product could interfere with endogenous fibrinolytic system which might result in higher risk of coronary artery stenosis [25,26]. Future experimental and clinical studies are warranted to investigate whether reduced $\mathrm{HbA1c}$ level will improve atherosclerosis plaque stability.

Other than the significant association between HbA1c level and the severity of CAD, our current study also showed that increased $\mathrm{HbA1c}$ level was associated with more vessel stenoses in non-diabetic patients. As 
aforementioned, by means of increasing vascular permeability, promoting endothelium dysfunction, and enhancing inflammation and oxidative reaction, increased advanced glycosylation end-product might lead to more vessels stenoses.

Consistent with previous some epidemiological studies $[11,18,19,27]$, data from our study also revealed that after adjusted for traditional risk factors including age, smoking, TC, HDL-C, HbA1c remained strongly associated with the severity of CAD. Notably, even after adjusted for fasting blood glucose, an independent risk factor for ASCVD and diabetes, there was still significant relationship between HbA1c and the severity of CAD, further supporting previous findings that $\mathrm{HbA1c}$ might be superior to fasting blood glucose in the respects of CVD risk discrimination. However, there was one distinctive discrepancy between our research and previous studies $[11,18,19,27]$ were that we concomitantly evaluated the relationship between HbA1c level and CAD severity including clinical scenario severity and the number of coronary artery stenosis.

As compared to fasting blood glucose, HbA1c level reflecting both fasting and post-prandial blood glucose might also partially explain the superiority of $\mathrm{HbA1c}$, because peak plasma glucose level might cause more severe damage to endothelium [28,29]. Future study is warranted to investigate whether there is significant interaction between post-prandial blood glucose and HbA1c on CVD risk prediction. Notably, there was no significant association between HbA1c and the severity of CAD when adjusted for CRP. The underlying mechanism might be ascribed to the increased CRP release when advanced glycosylated end-product was elevated.

\section{Conclusion}

Our observational study found out that in non-diabetic population, HbA1c level was significantly associated with the severity of coronary artery diseases, which further supporting the notion that HbA1c may be a useful and independent indicator for CVD risk evaluation.

\section{Competing interests}

The authors declare that they have no competing interests.

\section{Authors' contributions}

$A C, G L, X W$ and $X L$ performed this study, JC performed statistic analyses, $Y Z$ and $L L$ designed this study, and AC wrote this article. All authors read approved the final manuscript.

\footnotetext{
Acknowledgements

This work was supported by the grants from the Technology Project Foundation of Guangdong Province, China (2009A030301004, 2011B031800021, 2011B061300034 and 2011B031800263), Guangdong Natural Science Foundation (S2012010009532), Guangdong Medical Research Foundation (B2011002), Cardiovascular medication grant of Guangdong Province (2011X25), Medical Scientific Research Grant of the Health Ministry of Guangdong province, China (B2011310 and A2012663).
}

Received: 18 September 2014 Accepted: 30 November 2014 Published: 4 December 2014

\section{References}

1. American Diabetes Association: Standards of medical care in diabetes-2014. Diabetes Care 2014, 37 Suppl 1:S14-S80.

2. Zamora-Ros R, Forouhi NG, Sharp SJ, Gonzalez CA, Buijsse B, Guevara M, van der Schouw YT, Amiano P, Boeing H, Bredsdorff L, Clavel-Chapelon F, Fagherazzi G, Feskens EJ, Franks PW, Grioni S, Katzke V, Key TJ, Khaw KT, Kuhn T, Masala G, Mattiello A, Molina-Montes E, Nilsson PM, Overvad K, Perquier F, Quiros JR, Romieu I, Sacerdote C, Scalbert A, Schulze M, et al: The association between dietary flavonoid and lignan intakes and incident type 2 diabetes in European populations: the EPIC-InterAct study. Diabetes Care 2013, 36:3961-3970.

3. Selvin $\mathrm{E}$, Crainiceanu CM, Brancati FL, Coresh J: Short-term variability in measures of glycemia and implications for the classification of diabetes. Arch Intern Med 2007, 167:1545-1551.

4. American Diabetes Association: Standards of medical care in diabetes-2009. Diabetes Care 2009, 32 Suppl 1:S13-S61.

5. Greenland P, Alpert JS, Beller GA, Benjamin EJ, Budoff MJ, Fayad ZA, Foster E, Hlatky MA, Hodgson JM, Kushner FG, Lauer MS, Shaw LJ, Smith SC Jr, Taylor AJ, Weintraub WS, Wenger NK, Jacobs AK, Smith SC Jr, Anderson JL, Albert N, Buller CE, Creager MA, Ettinger SM, Guyton RA, Halperin JL, Hochman JS, Kushner FG, Nishimura R, Ohman EM, Page RL, et al: 2010 ACCF/AHA guideline for assessment of cardiovascular risk in asymptomatic adults: a report of the American college of cardiology foundation/American heart association task force on practice guidelines. J Am Coll Cardiol 2010, 56:e50-e103.

6. Anderson TJ, Gregoire J, Hegele RA, Couture P, Mancini GB, McPherson R, Francis GA, Poirier P, Lau DC, Grover S, Genest J Jr, Carpentier AC, Dufour R, Gupta M, Ward R, Leiter LA, Lonn E, Ng DS, Pearson GJ, Yates GM, Stone JA, Ur E: 2012 update of the Canadian Cardiovascular Society guidelines for the diagnosis and treatment of dyslipidemia for the prevention of cardiovascular disease in the adult. Can J Cardiol 2013, 29:151-167.

7. Silbernagel G, Grammer TB, Winkelmann BR, Boehm BO, Marz W: Glycated hemoglobin predicts all-cause, cardiovascular, and cancer mortality in people without a history of diabetes undergoing coronary angiography. Diabetes Care 2011, 34:1355-1361.

8. Selvin E, Steffes MW, Zhu H, Matsushita K, Wagenknecht L, Pankow J, Coresh J, Brancati FL: Glycated hemoglobin, diabetes, and cardiovascular risk in nondiabetic adults. N Engl J Med 2010, 362:800-811.

9. Kramer CK, Araneta MR, Barrett-Connor E: A1C and diabetes diagnosis: the Rancho Bernardo study. Diabetes Care 2010, 33:101-103.

10. Sacks DB: The diagnosis of diabetes is changing: how implementation of hemoglobin A1c will impact clinical laboratories. Clin Chem 2009, 55:1612-1614.

11. Garg N, Moorthy N, Kapoor A, Tewari S, Kumar S, Sinha A, Shrivastava A, Goel PK: Hemoglobin A(1c) in nondiabetic patients: an independent predictor of coronary artery disease and its severity. Mayo Clin Proc 2014, 89:908-916.

12. Di Angelantonio E, Gao P, Khan H, Butterworth AS, Wormser D, Kaptoge $S$, Kondapally Seshasai SR, Thompson A, Sarwar N, Willeit P, Ridker PM, Barr EL, Khaw KT, Psaty BM, Brenner H, Balkau B, Dekker JM, Lawlor DA, Daimon M, Willeit J, Njolstad I, Nissinen A, Brunner EJ, Kuller LH, Price JF, Sundstrom J, Knuiman MW, Feskens EJ, Verschuren WM, Wald N, et al: Glycated hemoglobin measurement and prediction of cardiovascular disease. JAMA 2014, 311:1225-1233.

13. American Diabetes Association: Diagnosis and classification of diabetes mellitus. Diabetes Care 2010, 33 Suppl 1:S62-S69.

14. Seaquist ER, Anderson J, Childs B, Cryer P, Dagogo-Jack S, Fish L, Heller SR, Rodriguez H, Rosenzweig J, Vigersky R: Hypoglycemia and diabetes: a report of a workgroup of the American Diabetes Association and the Endocrine Society. Diabetes Care 2013, 36:1384-1395.

15. Pradhan AD, Rifai N, Buring JE, Ridker PM: Hemoglobin A1c predicts diabetes but not cardiovascular disease in nondiabetic women. Am J Med 2007, 120:720-727.

16. O'Sullivan CJ, Hynes N, Mahendran B, Andrews EJ, Avalos G, Tawfik S, Lowery A, Sultan S: Haemoglobin A1c ( $\mathrm{HbA1C})$ in non-diabetic and diabetic vascular patients. Is $\mathrm{HbA1C}$ an independent risk factor and predictor of adverse outcome. Eur J Vasc Endovasc Surg 2006, 32:188-197.

17. Go AS, Mozaffarian D, Roger VL, Benjamin EJ, Berry JD, Blaha MJ, Dai S, Ford ES, Fox CS, Franco S, Fullerton HJ, Gillespie C, Hailpern SM, Heit JA, Howard VJ, Huffman MD, Judd SE, Kissela BM, Kittner SJ, Lackland DT, Lichtman JH, Lisabeth LD, Mackey RH, Magid DJ, Marcus GM, Marelli A, Matchar DB, McGuire DK, 3rd Mohler ER, Moy CS, et al: Heart disease and stroke 
statistics-2014 update: a report from the American Heart Association. Circulation 2014, 129:e28-e292.

18. Ashraf H, Boroumand MA, Amirzadegan A, Talesh SA, Davoodi G: Hemoglobin A1C in non-diabetic patients: an independent predictor of coronary artery disease and its severity. Diabetes Res Clin Pract 2013, 102:225-232.

19. Ayhan SS, Tosun M, Ozturk S, Alcelik A, Ozlu MF, Erdem A, Erdem K, Erdem FH, Yazici M: Glycated haemoglobin is correlated with the severity of coronary artery disease independently of traditional risk factors in young patients. Endokrynol Pol 2012, 63:367-371.

20. Brownlee M, Cerami A, Vlassara H: Advanced products of nonenzymatic glycosylation and the pathogenesis of diabetic vascular disease. Diabetes Metab Rev 1988, 4:437-451.

21. Brownlee M: The pathobiology of diabetic complications: a unifying mechanism. Diabetes 2005, 54:1615-1625.

22. Nathan DM, Cleary PA, Backlund JY, Genuth SM, Lachin JM, Orchard TJ, Raskin P, Zinman B: Intensive diabetes treatment and cardiovascular disease in patients with type 1 diabetes. N Engl J Med 2005, 353:2643-2653.

23. Geluk CA, Post WJ, Hillege HL, Tio RA, Tijssen JG, van Dijk RB, Dijk WA Bakker SJ, de Jong PE, van Gilst WH, Zijlstra F: C-reactive protein and angiographic characteristics of stable and unstable coronary artery disease: data from the prospective PREVEND cohort. Atherosclerosis 2008, 196:372-382.

24. Inoue T, Kato T, Uchida T, Sakuma M, Nakajima A, Shibazaki M, Imoto Y, Saito M, Hashimoto S, Hikichi Y, Node K: Local release of C-reactive protein from vulnerable plaque or coronary arterial wall injured by stenting. J Am Coll Cardiol 2005, 46:239-245.

25. Dunn EJ, Philippou H, Ariens RA, Grant PJ: Molecular mechanisms involved in the resistance of fibrin to clot lysis by plasmin in subjects with type 2 diabetes mellitus. Diabetologia 2006, 49:1071-1080.

26. Dunn EJ, Ariens RA, Grant PJ: The influence of type 2 diabetes on fibrin structure and function. Diabetologia 2005, 48:1198-1206.

27. Silbernagel G, Kleber ME, Grammer TB, Winkelmann BR, Boehm BO, Marz W: Additional use of glycated hemoglobin for diagnosis of type 2 diabetes in people undergoing coronary angiography reveals a subgroup at increased cardiovascular risk. Diabetes Care 2011, 34:2471-2473.

28. Monnier L, Lapinski H, Colette C: Contributions of fasting and postprandial plasma glucose increments to the overall diurnal hyperglycemia of type 2 diabetic patients: variations with increasing levels of $\mathrm{HbA}(1 \mathrm{c})$. Diabetes Care 2003, 26:881-885.

29. Diedrichs H, Pfister R, Clement Z, Hagemeister J, Schneider CA: Delta-glycated hemoglobin: a novel independent risk factor for cardiovascular events in patients without diabetes mellitus. J Endocrinol Invest 2009, 32:564-567.

doi:10.1186/1476-511X-13-181

Cite this article as: Cai et al:: Glycated hemoglobin level is significantly associated with the severity of coronary artery disease in non-diabetic adults. Lipids in Health and Disease 2014 13:181.

\section{Submit your next manuscript to BioMed Central and take full advantage of:}

- Convenient online submission

- Thorough peer review

- No space constraints or color figure charges

- Immediate publication on acceptance

- Inclusion in PubMed, CAS, Scopus and Google Scholar

- Research which is freely available for redistribution 